\title{
PROPÓSITOS DE LA EDUCACIÓN FRENTE A DESARROLLOS DE INTELIGENCIA ARTIFICIAL
}

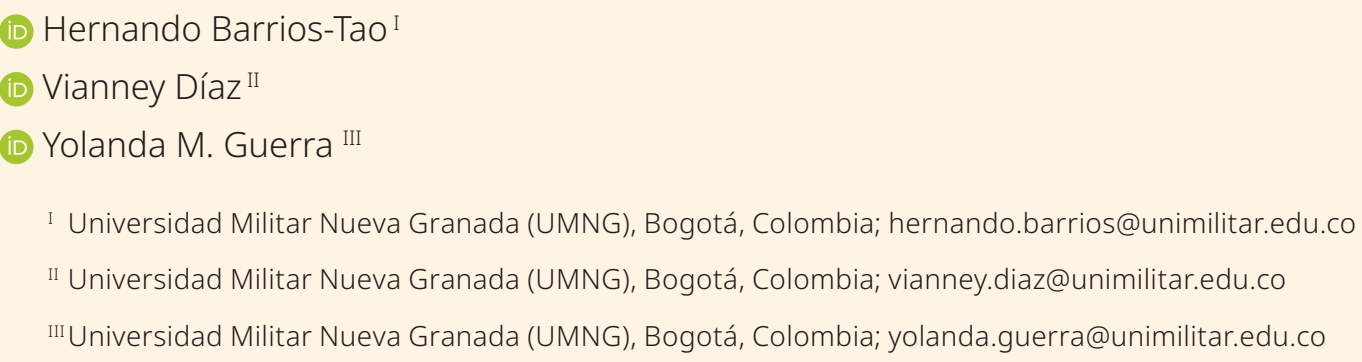

\section{Resumen}

La inteligencia artificial reconfigura entornos sociales, prácticas educativas y vidas humanas, y sus desarrollos movilizan voces defensoras y detractoras. El objetivo es interpretar posibilidades, riesgos y oportunidades de la inteligencia artificial para los propósitos de la educación, mediante una hermenéutica analógica que establezca un equilibrio entre dos extremos interpretativos: univocidad y equivocidad. Los resultados indican impactos de la inteligencia artificial en la visión humanista, la autonomía, la equidad, la formación integral y el mismo derecho a la educación. Una comprensión equilibrada mitiga riesgos y aprovecha posibilidades de estos desarrollos para los propósitos educativos, con la visión de que aún están en proceso y demandan nuevas indagaciones.

EDUCACIÓN • INTELIGENCIA ARTIFICIAL • PROCESO DE ENSEÑANZA-APRENDIZAJE • HUMANISMO

\section{PROPÓSITOS DA EDUCAÇÃO PERANTE OS DESENVOLVIMENTOS DE INTELIGÊNCIA ARTIFICIAL}

\section{Resumo}

A inteligência artificial reconfigura entornos sociais, práticas educacionais e vidas humanas, e seus desenvolvimentos mobilizam vozes defensoras e detratoras. O objetivo é interpretar possibilidades, riscos e oportunidades da inteligência artificial para os propósitos da educação, mediante uma hermenêutica analógica que estabeleça um equilíbrio entre dois extremos interpretativos: univocidade e equivocidade. Os resultados indicam impactos da inteligência artificial na visão humanista, a autonomia, a equidade, a formação integral e o próprio direito à educação. Uma compreensão equilibrada mitiga riscos e aproveita possibilidades desses desenvolvimentos para os propósitos educacionais, com a visão de que ainda estão em processamento e demandam novas indagações. 


\title{
PURPOSES OF EDUCATION ALONG WITH ARTIFICIAL INTELLIGENCE
} DEVELOPMENTS

\begin{abstract}
Artificial intelligence reconfigures social environments, educational practices and human lives, and its developments mobilize voices for advocates and detractors. The objective is to interpret possibilities, risks and opportunities of artificial intelligence for the purposes of education, through an analogical hermeneutic that establishes a balance between two interpretative extremes: univocity and equivocation. The results indicate impacts of artificial intelligence on the humanistic vision, autonomy, equity, comprehensive training and the same right to education. A balanced understanding mitigates risks and takes advantage of the possibilities of these developments for educational purposes, with the view that they are still in process and demand new inquiries.
\end{abstract}

EDUCATION • ARTIFICIAL INTELLIGENCE • TEACHING-LEARNING PROCESS • HUMANISM

\section{LES OBJECTIFS DE L'ÉDUCATION ET LE DÉVELOPPEMENT DE L'INTELLIGENCE ARTIFICIELLE}

\section{Résumé}

Des politiques de discrimination positive ont été mises en œuvre à l'Universidade Federal du Minas Gerais (UFMG), notamments les bonus et les quotas, afin de réduire les inégalités d'accès à l'enseignement supérieur. En outre, le Programa de Apoio a Planos de Reestruturação e Expansão das Universidades (Reuni) et l'utilisation de l'Exame Nacional do Ensino Médio/Sistema de Seleção Unificado (Enem/SiSU) dans le processus de sélection peuvent avoir contribué à augmenter la proportion de minorités dans les établissements publics d'enseignement supérieur au Brésil. L'objectif principal de ce travail est de vérifier l'évolution de la diversité du corps étudiant à l'UFMG entre 2006 et 2015, en abordant les effets de ces changements. Les politiques du Reuni, des bonus et des quotas semblent être efficaces pour réduire les inégalités de différents ordres à l'UFMG, alors que l'Enem/SiSU semble avoir favorisé une augmentation des inégalités. Par ailleurs ont été évalués les déterminants liés à l'appartenance à des ménages à faible revenu, au fait d'être noir ou d'avoir fréquenté des écoles secondaires privées ou publiques.

ÉDUCATION • INTELLIGENCE ARTIFICIELLE • PROCESSUS ENSEIGNEMENT-APPRENTISSAGE • HUMANISME 
OS SISTEMAS DE INTELIGENCIA ARTIFICIAL (IA) SUPERARON LA CONDICIÓN DE INSTRUMENTOS, irrumpieron en ámbitos otrora exclusivos del actuar humano, emulan acciones del ser humano y caminan en su reconfiguración y sustitución (Barrios-Tao et al., 2020). La IA es una "fuerza poderosa" que remodela entornos, culturas y relaciones humanas (Floridi et al., 2018; Marín, 2018; Pedró, 2019) y continúa con innovaciones: robots sociales, telepresencia colaborativa y almacenamiento de datos en ADN (World Economic Forum [WEF], 2019; Perez et al., 2017).

Estos desarrollos plantean desafíos y organismos, como la Organización de las Naciones Unidas para la educación, la ciencia y la cultura (Unesco), indican políticas en relación con sus riesgos y oportunidades para el desarrollo sostenible: garantizar inclusión y equidad, preparar los actores educativos, comprender las prácticas educativas y asegurar la ética en el manejo de datos (Pedró, 2019). Asimismo, la Unesco (2019a, 2019b) abrió escenarios para deliberar sobre el modo como la IA podría fortalecer la educación, mejorar el aprendizaje y promover el desarrollo de habilidades computacionales, y para formular preguntas en torno de sus límites y elementos para su humanización. Con este mismo objetivo, interacción humanismo-IA, la Universidad de Standford creó el Centro de IA centrado en el ser humano.

No obstante, los resultados de la revisión sistemática sobre aplicaciones de la IA en educación de Zawacki et al. (2019) indican una ausencia en este diálogo educación-IA: “¿Dónde están los educadores?” Esta pregunta inicial se traduce en preocupaciones sobre su falta de claridad acerca de la IA en sus prácticas de enseñanza-aprendizaje y sus alcances para fortalecer la educación: entre 145 artículos (de 2.656 entre 2007-2018), 13 (8,9\%) fueron escritos por primeros autores con formación en educación.

La relación de desarrollos de IA y educación se mueve entre tecnofílicos y tecnofóbicos, detractores y defensores. Posibilidades y riesgos generan valoraciones encontradas: entusiasmo por sus avances y temor por sus resultados (Perez et al., 2017); sueños por una "visión utópica" en sus procesos y pesadillas por un "final distópico" en su consecución (Martorell \& Alonso, 2019); interacciones "constructivas" que promueven valores para mejorar entornos y "destructivas" que provoquen afectaciones por los intereses económicos incubados (Toboso \& Aparicio, 2019); posibilidades para sus entornos sociales y riesgos para el desarrollo sostenible (Pedró, 2019); avances y potenciales riesgos (Sichman, 2021).

Frente a esta disparidad y antagonismo es necesario un equilibrio mediante una visión integral de sus desarrollos (Martorell \& Alonso, 2019) y una orientación sobre sus beneficios y sus posibles impactos negativos (Perez et al., 2017). Con el propósito de ofrecer una visión integral de la relación IA y educación, el objetivo del artículo es interpretar los beneficios, riesgos y oportunidades de los desarrollos de la IA para los propósitos de la educación.

\section{IA y propósitos de la educación}

El constructo IA se complejiza con la dinámica de sus desarrollos. De la pregunta inicial sobre la posibilidad de que una máquina fuese inteligente, se siguió a la emulación de voces y emociones, hasta los sueños de superar lo bumano (Kaplan \& Haenlein, 2019; Floridi et al., 2018; Postigo, 2018; García, 2017). El horizonte cronológico permite agrupar diversas concepciones y modos para clasificarla.

El primer grupo conceptual la ubica en un área multidisciplinaria (matemáticas, informática, psicología, sociología, economía, ingeniería, neurociencias, biología, estadística) con influencia de 
otros saberes (filosofía, lingüística) (Vicari, 2021; Stone, 2016; Zawacki et al., 2019). Una segunda concepción focaliza la relación sistemas-tecnologías y la define como una disciplina "relacionada con la teoría de la computación cuyo objetivo es emular algunas de las facultades intelectuales humanas en sistemas artificiales" (Benítez et al., 2014). El tercer concepto determina la actividad de crear máquinas inteligentes, en el sentido de una "cualidad" que les permite funcionar de manera apropiada y con previsión de su entorno (Stone, 2016). Un cuarto grupo supera el singular IA y caracteriza estos sistemas. Mientras que Luckin et al. (2016) los determinan como diseños para interactuar con su entorno a través de capacidades inteligentes, la Comisión Europea (2018, p. 2) los caracteriza con capacidades para “. . . analizar su entorno y pasar a la acción -con cierto grado de autonomía - con el fin de alcanzar objetivos específicos”. Allí se incluyen programas (asistentes de voz, de análisis de imágenes y reconocimiento facial, motores de búsqueda) y software incorporados en dispositivos de hardware (robots, drones o aplicaciones de la internet de las cosas).

En cuanto a su clasificación, se diferencia entre IA estrecha y general (Baker \& Smith, 2019), se señalan etapas: estrecha, general y super IA (Kaplan \& Haenlein, 2019), y se distingue entre versión débil y fuerte (Strong AI) (Zawacki et al., 2019; Navas, 2016). La versión fuerte ubica sistemas que afrontan "... desafíos filosóficos fuertes como la conciencia, el desarrollo de un carácter autónomo y único, o la sensibilidad, la empatía o la compasión" (Colmenarejo, 2018, p. 123). Kaplan y Haenlein (2019) clasifican estos sistemas de acuerdo con sus competencias: IA analítica con características de inteligencia cognitiva, IA inspirada en seres humanos con emociones para toma de decisiones e IA bumanizada con inteligencia social.

En el ámbito educativo, los sistemas de IA impactan actores educativos, prácticas y escenarios (Barrios-Tao et al., 2019). Además, ofrecen intervenciones en procesos académicos de los estudiantes: enganche, motivación, participación, retención, cognición (Zawacki et al., 2019; Marín, 2018; Huang, 2018). Asimismo, influyen en el rendimiento con tutores, aplicaciones, agentes autónomos inteligentes o asistentes virtuales (Sichman, 2021; Vicari, 2021; Alexander et al., 2019; Lodhi et al., 2018; Hussain et al., 2018; Howard et al., 2017; Sebastian \& Richards, 2017; Huang \& Chen, 2016) y reconfiguran escenarios mediante desarrollos de Realidad Aumentada (RA) que enriquecen los espacios físicos reales por medio de sonidos e imágenes (Munnerley et al., 2014) y Realidad Mixta (RM) en la cual coexisten objetos digitales y físicos (Adams et al., 2018). Por último, otros sistemas automatizan prácticas de enseñanza-aprendizaje con técnicas como la Logistic Regression (LR), Random Forest (RF), Data Mining (DM) entre otras (Sreenivasa et al., 2018; Umer et al., 2017; Hoffait \& Schyns, 2017).

Los impactos de estos desarrollos son un desafío fluctuante entre posibilidades, riesgos y oportunidades para los propósitos de la educación. En síntesis, se considera la educación como un derecho (Declaración de los Derechos Humanos, arts. 26 y 27), cuya centralidad es el desarrollo integral del ser humano mediante sus pilares del ser, conocer, hacer y convivir (Delors, 1996), que lo conduzcan por caminos de autonomía y libertad, para que contribuya, con procesos de inclusión y equidad, con la transformación sociocultural y con el desarrollo humano y social sostenible (Unesco, 2015).

\section{Metodología}

La metodología se ubica en la hermenéutica analógica de Beuchot: “... teoría de la interpretación de textos, que usa el concepto de analogía para estructurarse” (2008, p. 491). Esta "política de interpretación" aplicable a problemas sociales permite establecer un equilibrio entre dos extremos interpretativos, univocidad y equivocidad (Beuchot, 2016), situación de los textos interpretados, con relación a beneficios, riesgos y oportunidades de la IA para la educación.

El diseño metodológico se estructura con base en las tres fases de la hermenéutica analógica: sintáctica, semántica y pragmática (Beuchot, 2008), las cuales orientaron las acciones realizadas: 
1) Selección y revisión de textos de acuerdo con el objetivo, a partir de palabras claves en castellano, francés e inglés ("inteligencia artificial", "educación", "humanismo"), con los criterios de inclusión (artículos de investigación ubicados en bases de datos y sistemas: Science Direct, SciELO, EBSCO, Dialnet, Proquest; informes, reportes y declaraciones sobre IA y educación de universidades y entidades nacionales e internacionales; textos en inglés, francés, portugués y castellano; marco temporal: 2015-2020) y de exclusión (material gris; trabajos de maestría y tesis doctorales; idiomas diferentes); 2) Construcción de matriz para organizar los textos seleccionados, a partir de unidades temáticas determinadas como "propósitos" de la educación; 3) Caracterización de voces unívocas (beneficios) y de las equívocas (riesgos) de la IA para los propósitos de la educación. 4) La última acción corresponde con la fase pragmática, en la cual se determina la comprensión analógica $(A)$ o la phrónesis entre las voces unívocas $(U)$ y aquellas equívocas $(E)$. Este momento interpretativo permitió la determinación de temáticas emergentes en la relación IA y propósitos de la educación, base para la interpretación analógica: IA y derecho a la educación (1D), IA y humanismo (2H), IA y autonomía (3A), IA y equidad (4I), IA y formación integral (5F).

\section{Resultados}

\section{IA y derecho a la educación (U1D): entre mercantilismo (E1D) y cobertura (A1D)}

En los primeros textos con voces unívocas (U1D), unas investigaciones implementan sistemas de IA para prevenir problemas como la deserción y el bajo rendimiento que configurarían fracasos educativos (Rovira et al., 2017; Umer et al., 2017), otras crean desarrollos mediante minería de datos y algoritmos de aprendizaje automático que predicen, a partir de la motivación inicial de los estudiantes, tanto sus intenciones de abandono, como sus posibilidades de permanencia y éxito. De ahí resultan acciones de orientación profesional y de tutorías para reducir tasas de deserción (Sreenivasa et al., 2018; Hoffait \& Schyns, 2017). Con relación a esta ayuda estudiantil, Lynch (2018) se refiere a los "compañeros de aprendizaje permanente", que permiten conocer sus historias personales y escolares, así como reforzar habilidades y dominio de contenidos de aprendizaje.

Por otra parte, se presentan modelos basados en la minería de datos como la regresión logística, mediante árboles de decisión y redes neuronales, para predecir la retención estudiantil y posibilidades de graduación (Raju \& Schumacker, 2015). Asimismo, sistemas interactivos como el Intelligent Student Assistant (StuA) que asiste en la inducción estudiantil al ámbito universitario (Lodhi et al., 2018), los Embodied Conversational Agents (ECA) como apoyo en decidir y cambiar actitudes ante situaciones estigmatizantes construidas culturalmente (Sebastian \& Richards, 2017), y los Intelligent Tutoring Systems (ITS), basados en modelos de aprendizaje y redes neuronales, que indican rutas de aprendizaje, proporcionan una estructura cognitiva e involucran al estudiante con su aprendizaje (Zawacki et al., 2019).

Otros sistemas favorecen la inserción en el mundo laboral de los egresados como el Modelo Lingüistico que captura datos del currículum, fortalece la toma de decisiones con múltiples criterios y mejora solicitudes de prácticas en el mercado laboral, de acuerdo con sus preferencias (Nguyen et al., 2018). Asimismo, los sistemas expertos permiten la selección del personal, a partir de sus habilidades personales, sociales y sus conocimientos técnicos. Estos sistemas fortalecen la integración entre las competencias definidas en los currículos y sus perfiles de egreso, con las necesidades de las empresas (Sánchez et al., 2016).

Los últimos autores consideran ampliar la cobertura en educación mediante desarrollos con RA y RM, que permiten arribar a situaciones, entornos y contextos, con dificultades de acceso físico. Estas nuevas realidades permiten el acceso de estudiantes en situaciones remotas a 
escenarios académicos, investigativos y culturales, para fortalecer sus procesos de enseñanzaaprendizaje e investigación (Craig \& Georgieva, 2018) e interactuar con agentes virtuales que actúen como facilitadores y acompañantes de los estudiantes (Zawacki et al., 2019).

La primera voz equívoca de los desarrollos de IA frente a la educación como derecho $(E 1 D)$ se relaciona con factores comerciales, mercantilistas y lucrativos, influenciados por políticas neoliberales, que impactan actores y prácticas educativas. Mientras que Saura y Bolívar (2019, p. 11) distinguen dos perspectivas neoliberales en la educación: una basada en los "enfoques de condición estructural de las políticas" y otra "focalizada en aspectos de la nueva gestión pública”, Campos y Lastória (2020) retoman la conversión de la formación (bildung) a una "semiformación" (balbbildung), conquistada por el fetichismo de la mercancía, planteada por Adorno, y la conectan con la IA, la racionalidad instrumental y el aprendizaje.

Esta situación mercantilista se alimenta del valor comercial de datos y conocimiento, cuyo beneficio financiero se enruta hacia terceros. El valor de los datos vigilados es cada vez mayor para empresas que investigan sobre hábitos y preferencias de los usuarios. En el ámbito académico, Lupton (2016) señala las recopilaciones automáticas de publicaciones de software como Web of Science y Google Scholar que diseñan métricas sobre su desempeño y en las cuales “. . . los académicos no tienen opción sobre si desean o no que sus registros de publicación y citas se muestren públicamente en estas plataformas"1 (p. 110, traducción propia).

Otros autores indican que desarrollos de IA, a la vez que influenciaron el comercio en la educación, condujeron sus prácticas por el mercado de los rankings, la métrica de sus resultados y la medición productiva de los educadores. Este comercio de la producción de conocimiento, según Feldman y Sandoval (2018), creó dos niveles investigación. Una vinculada con la carrera profesional, con reconocimiento institucional, incentivos y transferencia de conocimiento, y otra desligada de la producción mercantil y afín con una acción ética relevante con la mejora de la sociedad y la formación ciudadana. Una mercantilista, recompensada por las disciplinas, y otra destacada desde el punto de vista social y humano.

En este marco productivo se limita el derecho a la educación a quienes tienen posibilidades monetarias y se configura la biometría de los sujetos académicos: "metrificados" (Beer, 2016), "datificados" (Cheney, 2017), "cuantificados" (Lupton, 2016), “digitalizados” (Moore \& Robinson, 2016). En cada denominación subyacen políticas neoliberales que ejercen una gobernanza suave mediante evaluaciones cuantitativas que desvían los propósitos de la educación (Saura \& Bolívar, 2019). Estudios señalan impactos bibliométricos que miden la calidad de las investigaciones mediante cuantificaciones digitalizadas y establecen valores comerciales a estos productos de conocimiento (Feldman \& Sandoval, 2018; Lupton et al., 2018).

Esta métrica, además de ser factor de capital y comercio, actúa como control que monitorea el supuesto valor y el comportamiento de las personas (Saura \& Bolívar, 2019). Así, la vida humana se convirtió en producto con valor monetario, “. . la la capitalización del cuerpo humano" (Lupton, 2016), por medio del precio establecido para los conocimientos biodigitales generados por las personas. En consecuencia, los actores educativos se convierten en prosumidores (productor-consumidor a la vez) y su información, datos y conocimiento en mercancía (Feldman \& Sandoval, 2018). Frente a la tecnología y su productividad, el prosumidor debe producir-consumir todo aquello producido-consumido. Allí, el actor educativo "se disuelve en la contracción del intervalo entre producción y consumo" y su reconocimiento se limita a su "respuesta funcional" exigida por el aparato productivo (Marín, 2018, p. 189). 
La primera analogía $(A 1 D)$ se sustenta con criterios cuantitativos: cuanto mayor sea el desarrollo de sistemas de IA, mayor serán las oportunidades para la cobertura en educación: "Cuanto más amplio sea el conjunto de funcionamientos disponibles en una sociedad, más inclusiva será y ofrecerá mayores posibilidades de vida a las personas, y una libertad de elección mayor, favoreciendo la igualdad de oportunidades" (Toboso \& Aparicio, 2019, p. 172). Así, más oportunidades para afrontar problemas de deserción y ayudar a los estudiantes con tutores personalizados que guían y gestionan el aprendizaje, con el cuidado de que los profesores, con su presencia e interacción, coadyuven con el desarrollo de habilidades relacionadas con el ser y el convivir para una vida económica y social equilibrada (Popenici \& Kerr, 2017).

\section{IA y humanismo (U2H): entre reconfigurar (E2H) y fortalecer lo humano (A2H)}

Una mayor dedicación al cultivo de las dimensiones físicas, emocionales e intelectuales en camino hacia una "excelencia propia de nuestra humanidad" (Zovko \& Dillon, 2018, p. 554) es una perspectiva univocista $(U 2 \mathrm{H})$ que beneficiaría una visión humanista de la educación extendida a toda la vida (Pedró, 2019). Con estos desarrollos las personas “deberán seguir aprendiendo a lo largo de sus vidas", "los sistemas educativos tendrán que adaptarse a este nuevo paradigma” y se ofrecerán "mejores opciones de formación para todos" (Telefónica, 2018, p. 34). La automatización permitirá eximir a educadores de actividades rutinarias y administrativas para dedicarse a su desarrollo integral, así como al cuidado y acompañamiento personalizado de sus estudiantes (Lynch, 2018; Popenici \& Kerr, 2017).

Frente a la interacción entre humanos, fundamental para el desarrollo personal y social (Rivera \& Sánchez, 2018), unas voces equívocas $(E 2 H)$ señalan el riesgo de transgredirla con la sustitución de lo bumano por máquinas inteligentes. Unas prácticas automatizadas debilitarían tanto lo que significa ser humano, como el propósito educativo de ser "un medio para llegar a ser lo más humano posible, tanto como individuos de valor único e inalienable como miembros de la comunidad humana universal" (Zovko \& Dillon, 2018, p. 555).

A partir de considerar datos y algoritmos como "unidades epistemológicas hechas para tener una forma de representación” y fenómenos "políticos, normativos y éticos” (Prinsloo, 2017, p. 143) con poder de moldear la vida social en varios grados y sobre los cuales los individuos tienen, cada vez más, menos control (Monasterio, 2017), unos autores exponen el proceso de co-configuración y co-constitución entre ser humano y tecnologías como horizonte para la comprensión humana, con la consecuente formación de la mente y extensión del cuerpo (Ihde \& Malafouris, 2019; Marín, 2018; Menéndez, 2018).

La IA se encamina de la emulación del "lenguaje natural” (Wei \& Zhao, 2019), a desarrollar "agentes racionales" (Russell, 2017; Baker, 2019), reconocer e incorporar "emociones" (Imani \& Montazer, 2019; Craig \& Georgieva, 2018), hasta otorgarles "responsabilidades" y "personalidades" (Keating \& Nourbakhsh, 2018) y desarrollar "conciencia" o "sentido moral" en estos sistemas (Monasterio, 2017; Perez et al., 2017). En consecuencia, incidirían en lo orgánico para modificarlo, mejorarlo y re-crearlo (García, 2017) y cambiar la naturaleza "líquida, transformable y fluida" del ser humano, con las "consecuencias físicas, psíquicas, sociológicas y ecológicas para la vida, la salud y la integridad de las personas" (Postigo, 2018, p. 55). Cambiar el concepto de lo que significa ser humano (Telefónica, 2018), transformar su condición y naturaleza (Torcal, 2018) o erosionar la autodeterminación humana (Floridi et al., 2018) abren riesgos de que los seres humanos pueden ser redefinidos por la tecnología (Popenici \& Kerr, 2017).

En consecuencia, algunos sistemas buscan reemplazar y sustituir a profesores con criterios de rentabilidad: "la enseñanza es reemplazada por soluciones automatizadas de IA" (Popenici \& Kerr, 2017, p. 9). Un riesgo para la estabilidad laboral del personal, con implicaciones éticas en relación con los datos confidenciales y la privacidad de los actores educativos (Zawacki et al., 2019) y con 
inquietudes acerca de si los "costosos programas de investigación de IA en educación, los sistemas inteligentes de tutoría y la minería de datos" se podrían considerar "una mejor opción de inversión" que una educación con más o mejores profesores (Bayne, 2015, p. 459; Kaplan \& Haenlein, 2019). La misma denominación teacherbot crea una "confusión de límites" (Bayne, 2015) y reduce el rol de los profesores a motivadores del aprendizaje o facilitadores de aula (Lynch, 2018) o a la supervisión, evaluación y gestión del aprendizaje en línea (Popenici \& Kerr, 2017). Más aún, la concepción y práctica de estos sistemas emancipados de su condición instrumental configura e hipervincula el maestro humano como su "funcionario" (Marín, 2018).

Por último, autores señalan dos fuerzas ejerciendo su poder sobre la transformación humana y social: la educación busca mejorar su contexto sociocultural con unos valores humanos y los sistemas de IA con sus datos y algoritmos, configurados para influir, invadir y transformar la conducta humana con otros valores de tipo económico (Torcal, 2018). Prinsloo (2017) indica que a pesar de la creciente conciencia sobre cómo los algoritmos transforman la noción de subjetividad humana, el propósito de formar sujetos políticos se enfrenta con formas y métodos sutiles y automatizados que invaden los actores educativos.

La segunda analogía $(A 2 \mathrm{H})$ se refiere a una automatización que facilita la vida de las personas y puede estar "al servicio de la autodeterminación humana", "fomentar la cohesión social, sin socavar la dignidad humana” (Floridi et al., 2018, p. 694). El Manifiesto de Telefónica (2018) equilibra entre la actualización y flexibilidad de los sistemas educativos para adaptarse al cambio e incertidumbre de la IA, con la necesidad de "un enfoque humanístico que fomente el pensamiento crítico y creativo, así como una filosofía de aprendizaje continuo” (p. 14).

En cuanto a la relación teacherbot-profesor, Bayne (2015) señala algunos aspectos analógicos: explorar prácticas de enseñanza automatizada que integren dimensión humanista y apropiación tecnológica; explorar formas de integrar la presencia del profesor con la automatización y de trabajar juntos profesores-bots sin jerarquías ontológicas y en aras de la productividad. En este sentido, Pedró (2019) integra el cuidado de aspectos creativos, empáticos y socioemocionales por parte de los profesores, con el apoyo de la IA en el aprendizaje y en tareas cognitivas rutinarias. Asimismo, se acentúa la interacción entre actores educativos con la insustituible presencia del profesor (Lynch, 2018; Popenici \& Kerr, 2017), con la oportunidad de extender su rol en los nuevos escenarios mediante los nuevos sistemas, el aprendizaje automático y las nuevas metodologías de aprendizaje activo (Adams et al., 2018).

\section{IA y autonomía (U3A): frente a gobernanza (E3A) y responsabilidad personal (A3A)}

Autonomía, pensamiento crítico para una ciudadanía responsable (Nussbaum, 2010) y uso público de la razón (Cortina, 2013) son propósitos que se podrían fortalecer (U3A) con el empoderamiento a las personas, la descentralización del poder y la participación ciudadana que posibilitan las plataformas digitales y la internet (Zafra, 2018). La hiperinformación y comunicación facilitadas por la IA vigorizan la autonomía y "el desarrollo de una inteligencia resistente ante el poder de la autoridad y las tradiciones ciegas" (Nussbaum, 2010, p. 11), así como los procesos flexibles y multidisciplinarios de aprendizaje (Lobo, 2018).

En contraste, la tercera voz equívoca (E3A) indica el riesgo para la autonomía debido a la manipulación y gobernanza mediante la programación algorítmica de la información y los datos personales. Estas configuraciones influyen en las decisiones individuales con el fin de manipular resultados, modificar conductas y adoptar cierto tipo de comportamientos de manera inconsciente (Cotino, 2017), mediante la creación y extensión de corrientes emocionales negativas (Zafra, 2018), y conducir a su "área gris" donde datos automatizados extrapolan contextos y hechos reales (Adams et al., 2018). Por otra parte, estos dispositivos acrecientan la "desventaja social de los grupos marginados" 
que no tienen control ni conocimiento sobre cómo se analizan y emplean sus datos, y configuran una "autoridad algorítmica" que ejerce un dominio sobre unos "sujetos automatizados", cuyas decisiones las toman los codificadores como parte de una "agenda política conservadora" (Lupton, 2016, p. 118).

El paso de la manipulación a la gobernanza es tan sutil como las técnicas con las cuales se realiza. Saura y Bolívar (2019) señalan el automonitoreo, el autocontrol y la libertad engañosa de la seducción, por medio de suaves procesos de control que monitorean la vida misma, con el resultado de un "sujeto visible y visibilizado, pero vacío" (p. 22). Esta gobernanza traza una contradicción y paradoja a los individuos cuando los mueve entre "zombis e inteligentes", "animales listos y genios" y entre "gobernados por las causas del cerebro y el entorno que impiden la libertad" y "lúcidos y creativos en extremo" (Espinosa, 2018, p. 119).

La paradoja gobernanza-autonomía falsa se ubica en el "panóptico digital", donde el individuo exhibe y expone su interioridad a través de sus datos, con los cuales se manipula y gobierna. Allí, cada uno es "panóptico de sí mismo", configurado con sus propios datos y manipulado mediante la psicopolítica neoliberal, que busca agradar en lugar de someter, por medio de la seducción gustosa ejercida con emociones positivas (Han, 2014). Así, se declara la muerte al "tipo histórico de Sapiens" que a partir de la conciencia de su propia dimensión individual se considera autónomo, independiente y autodeterminado (Marín, 2018).

Con la falaz autonomía se arriesga la construcción de sujeto político, propósito educativo, con la consecuente anulación en la participación democrática. De ahí que la Declaración de Montréal (2018) prevenga que los sistemas de IA no deberían desarrollarse, ni utilizarse "para imponer un estilo de vida particular a las personas, ya sea directa o indirectamente, mediante la implementación de mecanismos de vigilancia y evaluación opresivos o de incentivos".

El siguiente riesgo es la difuminación de la responsabilidad individual: “¿Quién es responsable de las decisiones de un sistema de IA? ¿El diseñador del algoritmo, su creador, su distribuidor, el propietario final?" (Telefónica, 2018, p. 69). Tanto responsabilidad como autonomía se erosionan por la fuerza de la presión social configurada en las redes sociales: "La fuerza de lo que piensen los demás ha demostrado tener capacidad suficiente para modificar el comportamiento de los sujetos" (Lanier, 2018). La Declaración de Montréal (2018) alerta sobre la posibilidad de que desarrollos de IA disminuyan "la responsabilidad de los seres humanos cuando se deben tomar decisiones".

La tercera analogía $(A 3 \mathrm{~A})$ indica un equilibrio entre las tareas delegadas a los sistemas de IA y el cuidado de la autonomía personal, mediante la supervisión y orientación de las programaciones de esos sistemas (Floridi et al., 2018; Lynch, 2018). Por su parte, la Declaración de Montréal (2018) indica un equilibrio entre el uso de sistemas de IA y el respeto por la autonomía, con la posibilidad de "cumplir sus propios objetivos morales y su concepción de una vida que valga la pena vivir". Asimismo, considera oportuno mantener "la diversidad social y cultural" y el desarrollo humano personal en el ámbito educativo, cuando estos sistemas evitan restringir "las elecciones de estilo de vida personales" y "la homogeneización de la sociedad mediante la estandarización de comportamientos y opiniones".

\section{IA y equidad (U4I): frente a sistemas excluyentes (E4I) e inclusión social (A4I)}

La cuarta voz unívoca (U4I) indica contribuciones de la IA para la equidad en educación mediante la introducción de dispositivos interactivos que multipliquen entornos automatizados y se incluyan más estudiantes ubicados en lugares remotos (Toboso \& Aparicio, 2019). La conectividad posibilitaría nuevos modos de presencialidad con las formas de realidad (RA y RM) sostenidas por redes $5 \mathrm{G}$ en las que interactúan hombre-máquina y se recrean formas en que las personas experimentan el mundo (Adams et al., 2018). Con esta "realidad extendida", además de eliminar las distancias físicas, se aprovechan desarrollos como la simulación de vista de 360 grados de entorno, mediante imágenes 3D, que permite experimentar virtualmente problemáticas mundiales y generar acciones frente a ellas (Craig \& Georgieva, 2018). 
Asimismo, se indica la posibilidad de apoyar el aprendizaje en países en desarrollo, mediante la telepresencia colaborativa y con el apoyo de sistemas de tutoría inteligentes como el Computer Assisted Learning (Pedró, 2019; Nye, 2015), mejorar las capacidades de los actores educativos, mediante una "inteligencia humana aumentada por la IA", y "encontrar nuevas soluciones a viejos y nuevos problemas”, a partir de una distribución de los recursos más justa y eficiente (Floridi et al., 2018, p. 693).

Por último, se indica una "interacción constructiva" con la transformación cultural mediante la "robótica interactiva" orientada a fomentar y promover valores propios del entorno, deseables y compartidos por sus actores para la mejora de su contexto social (Toboso \& Aparicio, 2019). Así, se favorece la equidad de los sistemas educativos con el uso de datos (Pedró, 2019) y la adopción de principios de responsabilidad, capacidad de elección y transparencia en el desarrollo de plataformas equitativas y algoritmos responsables (Telefónica, 2018). De ahí que Stewart (2018) resalte “. . . excelentes oportunidades para que los países en desarrollo reduzcan la brecha digital y creen sociedades más inclusivas" (p. 23).

El primer contraste señala nuevas brechas de exclusión social, digital y robótica $(E 41)$, que reflejan desigualdades de "acceso y uso", así como de "oportunidades" (Toboso \& Aparicio, 2019). En los países en desarrollo estas grietas se amplían debido a sus deficiencias en número de dispositivos y en conectividad: en estos países más de la mitad de su población no está conectada (Telefónica, 2018). Los riesgos se indican tanto en rezagos en los procesos de enseñanzaaprendizaje (Zurutuza, 2018), como en la privación de nuevos escenarios mediante las realidades extendidas (Munnerley et al., 2014).

La revisión de Nye (2015) sintetiza obstáculos para introducir sistemas de IA en países en desarrollo: disponibilidad de hardware de TIC; disponibilidad eléctrica; confiabilidad de internet; costos de datos; formación en habilidades básicas de TIC; barrera de idioma; falta de contenido culturalmente apropiado. Además, Zurutuza (2018) indica que problemas sistémicos de desigualdad en la distribución de fuentes e información se deben a falta de infraestructura, contenidos inadecuados y prejuicios motivados por el origen étnico y cultural y, también, Adams et al. (2018) señalan otras barreras como la desproporción en el estatus socioeconómico, la inequidad de género y decisiones legislativas que obstaculizan el arribo de innovaciones.

En consecuencia, se amplía también la brecha entre instituciones educativas (Pedró, 2019; Beer, 2016) como un "producto clave de los sistemas de producción neoliberales" bajo los parámetros de la utilidad (Feldman \& Sandoval, 2018, p. 218), y con las políticas de parametrización y clasificación no diferenciadas. Asimismo, las prácticas de generación, recolección y análisis de datos subscriben diferentes clases y brechas entre personas, de acuerdo con sus medios y funciones: unos con medios para recolectar datos, otros con artefactos y experiencia para analizarlos y fijar las reglas para su acceso y utilidad, y los últimos quienes los generan y con mínimas posibilidades: "Se crean así nuevas jerarquías políticas, económicas y sociales ... un big data rico y un pobre big data” (Cotino, 2017, p. 138). La convergencia de la exclusión e inequidad se focaliza en los "nuevos parias" (Lerman, 2013) identificados con millones de personas que permanecen en la periferia de los grandes datos.

Por último, investigaciones evidencian discriminación e inequidad en la configuración de algoritmos para el acceso a la educación, con sesgos de género en la creación de perfiles (Sebastian \& Richards, 2017) y con formas de acoso de sus creadores que impactan las emociones personales (Craig \& Georgieva, 2018). En este ámbito, Stewart (2018, p. 23) se refiere a los datos y algoritmos para seleccionar características y configurar modelos con códigos que pueden integrar "prejuicios implícitos e inconscientes de sus creadores humanos".

La Declaración de Montréal (2018) y Floridi et al. (2018) abren la cuarta (A4I) analogía: contribuir con el fortalecimiento de una sociedad justa y equitativa mediante la garantía de acceso para 
todos al conocimiento, a los recursos fundamentales y a los desarrollos de IA. Otro equilibrio se traza entre la integración de estos desarrollos a la educación y la oportunidad que ofrecen a la transformación de la cultura con valores educativos como la inclusión y la justicia. Esta transformación indica un diseño de la denominada "infosfera" con desarrollos cuyos valores prioritarios sean formativos y se orienten a mejorar la sociedad (Floridi, 2018).

El cierre de esta analogía indica un acceso equitativo e inclusivo a desarrollos como la robótica telepresencial que permitiría a estudiantes con necesidades especiales para asistir a la clase, mantener la continuidad de su aprendizaje (Pedró, 2019), así como las oportunidades para el aprendizaje y la investigación mediante realidades aumentadas y mixtas, que permiten involucrar a los actores educativos en "una conversación por un mundo muy diferente, uno que pase de la era de la información a la era de la experiencia” (Craig \& Georgieva, 2018).

\section{IA y formación integral: (U5F) frente a superficialidad (E5F) y habilidades (A5F)}

Las últimas fuentes unívocas indican posibilidades para fortalecer otros pilares: conocer, hacer y convivir (U5F). Unos autores se centran en el potencial de la IA para innovar teorías y enfoques cognitivos con la centralidad del aprendizaje activo (Popenici \& Kerr, 2017), así como para aumentar y enriquecer el aula, con las consecuentes oportunidades de aprendizajes múltiples y flexibles (Munnerley et al., 2014). Pedró (2019) se refiere a beneficios para el aprendizaje colaborativo mediante sistemas de aprendizaje automático y procesamiento de texto, que posibilitan crear grupos de discusión asíncronos, interconectar actores educativos y monitorear la información sobre sus discusiones, y la guía de su participación y aprendizaje. Es el caso del colaborador, Computer Science, que monitorea el comportamiento colaborativo de los estudiantes, los conduce hacia una actitud más productiva mediante la detección de momentos en los cuales disminuyeron su participación y utiliza tácticas para integrarlos de nuevo (Howard et al., 2017).

Para el aprendizaje autónomo, la realidad ampliada permite al estudiante ejercer rol de cocreador de entornos mixtos y fortalecer su aprendizaje, a través de estímulos e interacciones con experiencias de realidad virtual, que inducen respuestas cerebrales similares a las producidas con eventos del mundo físico (Adams et al., 2018). Asimismo, el aprendizaje personalizado se fortalece con experiencias adaptativas mediante sistemas que monitorean su progreso, personalizan y modifican sus rutas de aprendizaje, a partir de los datos y con tecnologías analíticas. Estos sistemas de aprendizaje adaptativo se ajustan al nivel y tipo de contenido, de acuerdo con el logro de habilidades del estudiante (Adams et al., 2018), así como a los diversos estilos de aprendizaje y a las múltiples inteligencias, con el aumento de factores de interés, atracción y flexibilidad en tiempos y lugares (Munnerley et al., 2014). En el marco de los desarrollos para personalizar los procesos docentes (Campos \& Lastória, 2020), uno de estos sistemas, Intelligence Computer Assisted Instruction, se basa en la teoría de aprendizaje constructivo y establece un modelo cognitivo personalizado que determina el progreso y dominio de contenidos, además de analizar, decidir y construir una estrategia de aprendizaje adaptada al estudiante (Huang \& Chen, 2016).

Otro desarrollo de "simbiosis" entre humano-máquina, es el sistema cooperativo de Walsh et al. (2017) en el cual "aprendiz- ser humano" y "aprendiz-máquina” se benefician de un ambiente de aprendizaje eficaz y eficiente, con resultados cognitivos y afectivos, mediante el aprendizaje automático adaptado a los dos. En casos problemáticos, "aprendiz-máquina” presenta material correctivo para el progreso del ser humano. Otros sistemas fortalecen habilidades argumentativas para la escritura: mientras que el de puntualización automatizada analiza aspectos lingüísticos y estructurales de la escritura (Perin \& Lauterbach, 2018), el Sistema para la evaluación de argumentos identifica el nivel de argumentación en párrafos y sugiere recomendaciones para mejorar la argumentación en las formulaciones e informes finales de los proyectos (García et al., 2018). 
Finalmente, otras fuentes convergen en beneficios para el saber conocer y hacer en un mundo 4.0 o el pensamiento computacional. Yadav et al. (2016, p. 1052) se refieren a que mientras para la Royal Society (2012) el pensamiento computacional es un "proceso de reconocer aspectos de la computación" y "aplicar herramientas y técnicas informáticas para comprender y razonar sobre procesos y sistemas naturales y artificiales", Barr y Stephenson (2011) lo concretizan en nueve aspectos: recolección, análisis y representación de datos, descomposición de problemas, abstracción, algoritmos y procedimientos, automatización, paralelización y simulación. Para su fortalecimiento, se desarrollan las Learning Analytics, determinadas como disciplinas orientadas a mejorar procesos de enseñanza-aprendizaje, mediante análisis de datos y generación de estándares para caracterizar hábitos, predecir respuestas y proporcionar comentarios (Pedró, 2019), o como técnicas computacionales para intervenciones pedagógicas de apoyo al aprendizaje, mediante datos: captura, informe, predicción, actuación y refinación (Umer et al., 2017).

En contraste, voces equívocas indican riesgos para el saber conocer, hacer y convivir (E5F): con la automatización de información y contenidos, las prácticas de enseñanza-aprendizaje podrían reducirse a formas automáticas de entrega, control y evaluación de contenidos que cumplen con formatos y estándares (Popenici \& Kerr, 2017), condicionar habilidades de aprendizaje dentro y fuera del aula (Campos \& Lastória, 2020), en detrimento de la construcción de conocimiento que vincule entornos y experiencias de los actores educativos. Asimismo, se indican riesgos si se promocionan prácticas de enseñanza sin "evaluaciones rigurosas" (Pedró, 2019, p. 28) y tareas académicas e investigativas con base en la información superficial, automática e inmediata que ofrecen algunos sistemas.

Además, la automatización puede socavar la creatividad, pilar del saber hacer. Este riesgo es proporcional entre los roles delegados a los sistemas y aquellos disminuidos a los actores educativos, que deberían estar sujetos a la supervisión y elección humana (Floridi et al., 2018). La automatización de estas funciones obnubilaría el desarrollo de la "creatividad, la iniciativa y el espíritu emprendedor" (Popenici \& Kerr, 2017, p. 4), interferiría, redefiniría y diluiría las intenciones del artista en el proceso creativo (Venancio, 2019), reduciría la formación a competencias automatizadas, medibles y explotables por el mercado, y la educación a la producción y aumento de mano de obra: una tarea, una profesión y un medio orientado a un fin fiscal "donde la adquisición de conocimiento es un modo para la reproducción y el avance económico" (Feldman \& Sandoval, 2018, p. 218), en contravía de una educación centrada en lo que significa ser humano (Zovko \& Dillon, 2018).

Por último, se indican experiencias sin vínculos interpersonales incubadas por desarrollos tecnológicos que dificultan la formación del saber convivir, el fortalecimiento de valores comunitarios y el desarrollo de espacios comunes de experiencia (Marín, 2018). La dialéctica entre multitud-soledad, en la que divagan individuos, generó el fenómeno hikikomori, caracterizado por el abandono de interacciones sociales para refugiarse en tecnologías, a través de las cuales se establece una relación virtual con el mundo exterior (De la Calle Real \& Muñoz Algar, 2018; Sánchez, 2017). En este caso, interacción, crecimiento y convivencia entre actores educativos está en riesgo, ya que ahora se presenta sólo una coexistencia entre "entes fantasmagóricos" (Lanier, 2018) o entre perfiles ficticios, creados para mostrar lo que no se es, que redundan en las plataformas. Crece el aislamiento y la soledad, en detrimento de comunidades de aprendizaje, necesarias para el desarrollo en los ámbitos educativos.

La última analogía traza un equilibrio entre fortalecer el pensamiento computacional y automatizar unas prácticas educativas $(A 5 \mathrm{H})$. Walsh et al. (2017) señalan la interacción máquina-ser humano con enfoques multidisciplinarios, que permiten un equilibrio entre el aprendizaje humano y los impactos del aprendizaje automático en el estudiante. Esta "sinfonía del sistema" necesita una integración y evaluación simultánea entre teorías de las ciencias cognitivas, educación y ciencias de la computación. Así, para una equilibrada interacción máquina-ser humano o smart-intelligence es 
prioritario fortalecer la inteligencia humana en la toma de decisiones y las habilidades computacionales, con potencialidades de sistemas de IA (Baker, 2019).

\section{Discusión}

El primer aspecto para discutir es la apuesta por una educación humanista que integre los desarrollos de la IA, no reducidos a una "solución centrada en la tecnología", sino que considere su utilidad para hacernos más humanos (Popenici \& Kerr, 2017), que afiance su dimensión sapiencial para profundizar el sentido de lo humano (Floridi, 2018; Postigo, 2018) y que cuide la sostenibilidad humana y social con garantía de acceso y equidad.

Un segundo punto se traza como necesidad: la comprensión mutua entre educación y desarrollos de IA. Se debe iniciar con el reconocimiento mutuo de posibilidades, seguir con los aspectos que deberían ser fortalecidos para mejorar su relación y los riesgos que podrían afectarlas, y culminar con las potencialidades que deberían confluir en el bienestar integral, la calidad de vida de las personas y sus entornos. Esta comprensión determinaría beneficios mutuos: la contribución de los sistemas de IA con el mejoramiento de la educación y las habilidades computacionales fortalecerían la creación de innovadores desarrollos de IA.

El tercer aspecto es un desafío. A quienes desarrollan sistemas de IA: un mayor conocimiento de lo humano y de las prácticas educativas orientadas al desarrollo integral, que superan información, recolección y análisis de datos. A los actores educativos: superar el "carácter de un consumo masivo en medio del capitalismo académico" (Brunner et al., 2019, p. 132), salir de la burbuja y repensar algunas de sus teorías y prácticas ancladas en el pasado y tímidas con el llamado a la innovación (Freire, 2017), fortalecer habilidades que no podrán ser sustituidas por máquinas y aprovechar mejor los desarrollos de IA.

Por último, se considera la integración educación-IA, humanismo-IA: articular prácticas y potencialidades. Una educación "a prueba de robots" (Aoun, 2017) no debe llenar las mentes con avances científicos, sino potenciar la mentalidad creativa y la elasticidad mental a fin de innovar en pro de la sociedad, para lo cual se deben integrar nuevas alfabetizaciones: de datos, para administrarlos; tecnológica, para saber cómo funcionan las máquinas; humana, humanics, para comprender lo que significa ser humano. El fin de educación-IA debería ser el bienestar integral de lo humano, un proceso revolucionario en la cotidianidad de los actores educativos apoyando sus objetivos y prácticas (Roll \& Wylie, 2016) con capacidad de no "fagocitar", sino "integrarse" con lo humano para constituirse como parte de él, buscar su crecimiento y dignificar sus dimensiones corporales, espirituales y sociales (Torcal, 2018).

\section{Conclusiones}

Los hallazgos indican posibilidades de que sistemas de IA fortalezcan propósitos de la educación, iniciando por una mayor garantía de acceso y equidad, viabilizada por sus innovadores desarrollos. Además, con la automatización, integración e interacción con sistemas de IA, se evidencian posibilidades de mejorar algunas prácticas educativas. No obstante, se alertan sobre riesgos para los objetivos de la educación, asociados con una mercantilización de la educación y sus consecuencias en términos de exclusión e inequidad, la posible manipulación y gobernanza que dificultaría procesos de autonomía y libertad, y el riesgo de la reconfiguración de lo bumano con valores oscuros, por parte de quienes configuran estos sistemas.

La posibilidad de una hermenéutica analógica con relación a los impactos de los sistemas de IA en las prácticas y en los actores educativos, todavía permanece abierta debido a que una IA completamente 
desarrollada aún no existe (Perez et al., 2017), y a que los alcances de esos desarrollos aún no se pueden medir totalmente en términos de beneficencia o maledicencia para lo bumano. De ahí que muchos autores e instituciones planteen más bien desafíos, algunos de los cuales pueden tomarse a modo de conclusiones: una política pública integral sobre IA para el desarrollo sostenible; garantizar la inclusión y la equidad en la IA en la educación; preparar a los docentes para una educación basada en IA y preparar la IA para comprender la educación; desarrollar sistemas de datos inclusivos y de calidad; hacer que la investigación sobre IA en la educación sea significativa (Pedró, 2019, p. 26).

Algunas de las fuentes se dirigen tanto a quienes desarrollan sistemas de IA como a los actores educativos para interactuar y aprovechar las oportunidades de estos desarrollos, a la vez que minimizar sus riesgos en pro de mejorar la vida humana y los ambientes sociales (Telefónica, 2018; Floridi et al., 2018). Asimismo, fomentar estos sistemas en beneficio del desarrollo humano y profesional de los actores educativos y evitar los extremos de sobre o infra utilizarlos, de infra o sobrevalorarlos.

A los objetivos de la educación se abren retos en la medida que avanzan innovaciones en los desarrollos de IA, los cuales abren el artículo a nuevas indagaciones: habilidades y destrezas blandas y sistemas de IA; teorías educativas y desarrollos de IA; sistemas de IA adaptados a entornos educativos particulares; configuración de valores humanísticos en sistemas de IA.

\section{Agradecimientos}

El artículo es producto del Proyecto de investigación INV-HUM-3183 “Tendencias temáticas y líneas de investigación de los MOOC en universidades acreditadas en Colombia”, financiado por la Vicerrectoría de Investigaciones de la Universidad Militar Nueva Granada, Colombia.

\section{Referencias}

Adams, S., Brown, M., Dahlstrom, E., Davis, A., Depaul, K., Diaz, V., \& Pomerantz, J. (2018). NMC Horizon Report: 2018 Higher Education Edition. EDUCAUSE.

Alexander, B., Ashford-Rowe, K., Barajas-Murph, N., Dobbin, G., Knott, J., Mccormack, M., Pomerantz, J., Seilhamer, R., \& Weber, N. (2019). EDUCAUSE Horizon Report 2019 Higher Education Edition. EDUCAUSE.

Aoun, J. (2017). Robot-Proof. Higher Education in the Age of Artificial Intelligence. MIT Press.

Baker, R. (2019). Stupid tutoring systems, intelligent humans. International Journal of Artificial Intelligence in Education, 26, 600-614.

Baker, T., \& Smith, L. (2019). Educ-AI-tion rebooted? Exploring the future of artificial intelligence in schools and colleges. Nesta.

Barr, V., \& Stephenson, C. (2011). Bringing computational thinking to K-12: What is involved and what is the role of the computer science education community? ACM Inroads, 2(1), 48-54.

Barrios-Tao, H., Díaz, V., \& Guerra, Y. (2019). Artificial intelligence and education, challenges and disadvantages for the teacher. ARCTIC Journal, 72(12), 30-50.

Barrios-Tao, H., Díaz, V., \& Guerra, Y. (2020). Subjetividades e inteligencia artificial: Desafíos para 'lo humano'. Veritas, 47(4), 81-107.

Bayne, S. (2015). Teacherbot: Interventions in automated teaching. Teaching in Higher Education, 20(4), 455-467.

Beer, D. (2016). Metric power. Palgrave Macmillan.

Benítez, R., Escudero, G., \& Kanaan, S. (2014). Inteligencia artificial avanzada. Universidad de Cataluña: UOC.

Beuchot, M. (2008). Breve exposición de la hermenéutica analógica. Revista Teología, 45(97), 491-502. 
Beuchot, M. (2016). Hechos e interpretaciones. Hacia una hermenéutica analógica. FCE.

Brunner, J., Labraña, J., Ganga, F., \& Rodríguez, E. (2019). Idea moderna de Universidad: De la torre de marfil al capitalismo académico. Educación XX1, 22(2), 119-140.

Campos, L. F., \& Lastória, L. (2020). Semiformação e inteligência artificial no ensino. Pro-Posiçôes, 31, e20180105.

Cheney, J. (2017). We are data: Algorithms and the making of our digital selves. University Press.

Colmenarejo, R. (2018). Ética aplicada a la gestión de datos masivos. Anales de la Cátedra Francisco Suárez, 52, 113-129.

Comisión Europea. (2018). Comunicación de la Comisión al Parlamento Europeo, al Consejo Europeo, al Consejo, al Comité Económico y Social Europeo y l Comité de las Regiones - Inteligencia artificial para Europa [COM(2018) 237]. https://ec.europa.eu/transparency/regdoc/rep/1/2018/ES/COM-2018237-F1-ES-MAIN-PART-1.PDF

Cortina, A. (2013). El futuro de las humanidades. Revista Chilena de Literatura, 84, 207-217.

Cotino, L. (2017). Big data e inteligencia artificial: Una aproximación a su tratamiento jurídico desde los derechos fundamentales. Dilemata, 24, 131-150.

Craig, E., \& Georgieva, M. (2018). VR and AR: The ethical challenges ahead. EDUCAUSE Review. https://er.educause.edu/blogs/2018/4/vr-and-ar-the-ethical-challenges-ahead

De la Calle, M., \& Muñoz, M. (2018). Hikikomori: El síndrome de aislamiento social y juvenil. Revista de la Asociación Española de Neuropsiquiatría, 38(133), 115-129.

Declaración de los Derechos Humanos. https://www.un.org/es/universal-declaration-human-rights/

Delors, J. (Ed.). (1996). La educación encierra un tesoro. Santillana.

Espinosa, L. (2018). La mano y el algoritmo: Una antropología compleja ante los desafíos tecnológicos del presente. Araucaria, 40, 109-136.

Feldman, Z., \& Sandoval, M. (2018). Metric power and the academic self: Neoliberalism, knowledge and resistance in the British university. TripleC, 16(1), 214-233.

Floridi, L. (2018). Soft ethics and the governance of the digital. Philosophy \& Technology, 31(4), 1-8.

Floridi, L., Cowls, J., Beltrametti, M., Chatila, R., Chazerand, P., Dignum, V., Luetge, Ch., Madelin, R., Pagallo, U., Rossi, F., Schafer, B., Valcke, P., \& Vayena, E. (2018). AI4People - An ethical framework for a good AI society: Opportunities, risks, principles, and recommendations. Minds and Machines, 28, 689-707.

Freire, J. (2017). El futuro de la educación: Aprender lo que no pueden hacer las máquinas. https://www.eldiario.es/sociedad/Educacion-Aprender-puedan-hacer-maquinas_0_723378320.html

García, J. (2017). ¿Cómo mejorar al ser humano? Un análisis de las tecnologías convergentes desde la antropología pedagógica. Pedagogia e Vita, 75, 94-105.

García, J., López, A., \& González, S. (2018). Automatic argument assessment of final project reports of computer engineering students. Computer Applications in Engineering Education, 26(5), 1217-1226.

Han, B.-Ch. (2014). Psicopolitica. Herder.

Hil, R. (2016). What an algorithm is? Philosophy \& Technology, 29(1), 35-59.

Hoffait, A., \& Schyns, M. (2017). Early detection of university students with potential difficulties. Decision Support Systems, 101, 1-11.

Howard, C., Jordan, P., Di Eugenio, B., \& Katz, S. (2017). Shifting the load: A peer dialogue agent that encourages its human collaborator to contribute more to problem solving. International Journal of Artificial Intelligence in Education, 27(1), 101-129.

Huang, J., \& Chen, Z. (2016). The research and design of web-based intelligent tutoring system. International Journal of Multimedia and Ubiquitous Engineering, 11(6), 337-348.

Huang, Sh. (2018). Effects of using artificial intelligence teaching system for environmental education on environmental knowledge and attitude. Eurasia Journal of Mathematics, Science and Technology Education, 14(7), 3277-3284. 
Hussain, M., Zhu, W., Zhang, W., \& Abidi, S. (2018). Student engagement predictions in an e-Learning system and their impact on student course assessment scores. Computational Intelligence and Neuroscience. https://doi.org/10.1155/2018/6347186

Ihde, D., \& Malafouris, L. (2019). Homo faber revisited: Postphenomenology and material engagement theory. Philosophy \& Technology, 32(2), 195-214.

Imani, M., \& Montazer, G. (2019). A survey of emotion recognition methods with emphasis on E-Learning environments. Journal of Network and Computer Applications, 147, 102423.

Kaplan, A., \& Haenlein, M. (2019). Siri, Siri, in my hand: Who's the fairest in the land? On the interpretations, illustrations, and implications of artificial intelligence. Business Horizons, 62, 15-25.

Keating, J., \& Nourbakhsh, I. (2018). Teaching artificial intelligence and humanity. Communications of the $A C M, 61(2), 29-32$.

Lanier, J. (2018). Diez razones para borrar tus redes sociales de inmediato. Debate.

Lerman, J. (2013). Big Data and Its Exclusions. Stanford Law Review, 66. https://www.stanfordlawreview. org/online/privacy-and-big-data-big-data-and-its-exclusions/

Lobo, L. C. (2018). Inteligência artificial, o futuro da medicina e a educação médica. Revista Brasileira de Educação Médica, 42(3), 3-8.

Lodhi, P., Mishra, O., Jain, S., \& Bajaj, V. (2018). StuA: An intelligent student assistant. International Journal of Interactive Multimedia and Artificial Intelligence, 5(2), 17-25.

Luckin, R., Holmes, W., Griffiths, M., \& Forcier, L. (2016). Intelligence Unleashed. An argument for AI in Education. Pearson.

Lupton, D. (2016). The diverse domains of quantified selves: Self-tracking modes and dataveillance. Economy and Society, 45(1), 101-122.

Lupton, D., Mewburn, I., \& Thomson, P. (2018). The digital academic: Critical perspectives on digital technologies in higher education. Routledge.

Lynch, M. (2018). 7 Roles for Artificial Intelligence in Education. https://www.thetechedvocate.org/7-roles-forartificial-intelligence-in-education/

Marín, J. (2018). La resemantización TIC de la cultura humanista. Index.Comunicación, 8(1), 179-195.

Martorell, F., \& Alonso, A. (2019). Asistidos por el soberano electrónico: Utopías y distopías de la inteligencia artificial. Dilemata, 30, 111-127.

Menéndez, A. (2018). Horizontes tecnológicos. Telos, 109, 10-15.

Monasterio, A. (2017). Ética algorítmica: Implicaciones éticas de una sociedad cada vez más gobernada por algoritmos. Dilemata, 24, 185-217.

Montréal Declaration for a Responsible Development of Artificial Intelligence. (2018). https://recherche.umontreal.ca/english/strategic-initiatives/montreal-declaration-for-a-responsible-ai/

Moore, Ph., \& Robinson, A. (2016). The quantified self: What counts in the neoliberal workplace. New Media \& Society, 18(11), 1-19.

Munnerley, D., Bacon, M., Fitzgerald, R., Wilson, A., Hedberg, J., Steele, J., \& Standley, A. (2014). Augmented Reality: Application in higher education. Australian Government. Office for Learning \& Teaching.

Navas, S. (2016). Smart robots y otras máquinas inteligentes en nuestra vida cotidiana. Revista CESCO de Derecho de Consumo, 20, 82-109.

Nguyen, J., Sánchez, G., Armisen, A., Agell, N., Rovira, X., \& Angulo, C. (2018). A linguistic multi-criteria decision-aiding system to support university career services. Applied Soft Computing Journal, 67, 933-940.

Nussbaum, M. (2010). Sin fines de lucro, por qué la democracia necesita de las humanidades. Katz.

Nye, B. (2015). Intelligent tutoring systems by and for the developing world. International Journal of Artificial Intelligence in Education, 25(2), 177-203. 
Organización de las Naciones Unidas para la Educación, la Ciencia y la Cultura. (2015). Replantear la educación: Hacia un bien común mundial. https://unesdoc.unesco.org/ark:/48223/pf0000232697

Organización de las Naciones Unidas para la Educación, la Ciencia y la Cultura. (2019a). Principios para la inteligencia artificial. ¿Hacia un enfoque humanista? https://es.unesco.org/news/unesco-celebraprimera-conferencia-mundial-promover-inteligencia-artificial-humanista

Organización de las Naciones Unidas para la Educación, la Ciencia y la Cultura. (2019b). Seis retos para aplicar la inteligencia artificial en educación. https://profuturo.education/profuturo-y-la-unescopresentan-los-seis-retos-para-aplicar-la-inteligencia-artificial-en-la-educacion/

Pedró, F. (Dir.) (2019). Artificial intelligence in education: Challenges and opportunities for sustainable development. Unesco. https://unesdoc.unesco.org/ark:/48223/pf0000366994

Perez, J. A., Deligianni, F., Ravi, D., \& Yang, G. (2017). Artificial Intelligence and Robotics. EPSRC UK-RAS Network. doi: 10.31256/WP2017.1. https://www.ukras.org/publications/white-papers/artificialintelligence-robotics/

Perin, D., \& Lauterbach, M. (2018). Assessing text-based writing of low-skilled college students. International Journal of Artificial Intelligence in Education, 28(1), 56-78.

Popenici, S., \& Kerr, Sh. (2017). Exploring the impact of artificial intelligence on teaching and learning in higher education. Research and Practice in Technology Enhanced Learning. https://doi.org/10.1186/s41039-017-0062-8

Postigo, E. (2018). La obsolescencia del ser humano. El advenimiento del hombre nuevo. Telos, 109, 51-59.

Prinsloo, P. (2017). Fleeing from Frankenstein's monster and meeting Kafka on the way: Algorithmic decision-making in higher education. E-Learning and Digital Media, 14(3), 138-163.

Raju, D., \& Schumacker, R. (2015). Exploring student characteristics of retention that lead to graduation in higher education using data mining models. Journal of College Student Retention: Research, Theory and Practice, 16(4), 563-591.

Rivera, J., \& Sánchez, D. (2018). Inteligencia artificial ¿Reemplazando al humano en la psicoterapia? Escritos, 24(53), 271-291.

Roll, I., \& Wylie, R. (2016). Evolution and revolution in artificial intelligence in education. International Journal of Artificial Intelligence in Education, 26(2), 582-599.

Rovira, S., Puertas, E., \& Igual, L. (2017). Data-driven system to predict academic grades and dropout. PLoS One, 12(2), 1-21.

Russell, S. (2017). Inteligencia artificial de beneficios probados. In AAVV. El próximo paso. La vida exponencial (pp. 175-192). OpenMind.

Sánchez, A. (2017). El fenómeno hikikomori: Tradición, educación y tecnologías de la información y la comunicación (TIC). Arbor, 193(785), 405.

Sánchez, L., Santos, A., Álvarez, E., Huerta, M., Camacho, S., \& Fernández, E. (2016). Development of an expert system for the evaluation of students' curricula on the basis of competencies. Future Internet, 8(2). https://doi.org/10.3390/fi8020022

Saura, G., \& Bolívar, A. (2019). Sujeto académico neoliberal: Cuantificado, digitalizado y bibliometrificado. Revista Iberoamericana sobre Calidad, Eficacia y Cambio en Educación - REICE, 17(4), 9-26.

Sebastian, J., \& Richards, D. (2017). Changing stigmatizing attitudes to mental health via education and contact with embodied conversational agents. Computers in Human Behavior, 73, 479-488.

Sichman, J. (2021). Inteligência Artificial e sociedade: avanços e riscos. Estudos Avançados, 35(101), 37-50.

Sreenivasa, K., Swapna, N., \& Praveen, P. (2018). Educational data mining for student placement prediction using machine learning algorithms. International Journal of Engineering and Technology, 7(1.2), 43-46.

Stewart, U. (2018). El potencial de la IA para reducir la brecha digital y crear una sociedad inclusiva. ITU News, 1, 19-23.

Stone, P. (Dir.) (2016). Artificial Intelligence and Life in 2030. One Hundred Year Study on Artificial Intelligence. Stanford University. https://ai100.stanford.edu/sites/g/files/sbiybj18871/files/media/ file/ai100report10032016fnl_singles.pdf 
Telefónica. (2018). Manifiesto por un nuevo pacto digital. Una digitalización centrada en las personas. https://www.telefonica.com/es/wp-content/uploads/sites/4/2021/06/manifiesto_por_un_nuevo_ pacto_digital.pdf

The Royal Society. (2012). Shut down or restart? The way forward for computing in UK schools. The Royal Society.

Toboso, M. \& Aparicio, M. (2019). Entornos de funcionamientos robotizados. ¿Es posible una robótica inclusiva? Dilemata, 11(30), 171-185.

Torcal, L. (2018). Integración versus superación. El corazón del problema tecnoético. Telos, 109, 60-69.

Umer, R., Susnjak, T., Mathrani, A., \& Suriadi, S. (2017). On predicting academic performance with process mining in learning analytics. Journal of Research in Innovative Teaching, 10(2), 160-176.

Venancio, S. J., Jr. (2019). Arte e inteligências artificiais: Implicações para a criatividade. Revista ARS, 17(35), 183-201.

Vicari, R. M. (2021). Influências das Tecnologias da Inteligência Artificial no ensino. Estudos Avançados, 35(101), 73-84.

Walsh, K., Hoque, M. \& Williams, K. (2017). Human machine learning symbiosis. Journal of Learning in Higher Education, 13(1), 55-62.

Wei, P., \& Zhao, Y. (2019). A novel speech emotion recognition algorithm based on wavelet kernel sparse classifier in stacked deep auto-encoder model. Personal and Ubiquitous Computing, 23, 521-529.

World Economic Forum. (2019). Top 10 Emerging Technologies. http://www3.weforum.org/docs/WEF_ Top_10_Emerging_Technologies_2019_Report.pdf

Yadav, A., Good, J., Voogt, J., \& Fisser, P. (2016). Computational Thinking as an Emerging Competence Domain. In M. Mulder (Ed.), Competence-Based Vocational and Professional Education: Bridging the Worlds of Work and Education (pp. 1051-1067). Springer International Publishing AG.

Zafra, J. (2018). Tenemos que cambiar el modelo: Toda la economía se basa en manipular personas. Entrevista a Jaron Lanier. Telos, 109, 26-34.

Zawacki, O., Marín, V., Bond, M., \& Gouverneur, F. (2019). Systematic review of research on artificial intelligence applications in higher education -Where are the educators? International Journal of Educational Technology in Higher Education, 16(39). https://doi.org/10.1186/s41239-019-0171-0

Zovko, M., \& Dillon, J. (2018). Humanism vs. competency: Traditional and contemporary models of education. Educational Philosophy and Theory, 50(6), 554-564.

Zurutuza, N. (2018). Pobreza de información e igualdad algorítmica: Poner los avances de la IA al alcance de las poblaciones más vulnerables. ITU News, 1, 26-28.

\section{Nota sobre la autoría}

Hernando Barrios-Tao: idea del artículo; revisión e interpretación analógica; escritura; discusión y edición final. Vianney Díaz: revisión y temáticas unívocas; matriz temática; escritura; discusión. Yolanda M. Guerra: revisión y temáticas equívocas; escritura; discusión y edición final.

\section{Disponibilidad de datos}

Los datos subyacentes al texto de la investigación se informan en el artículo.

\section{Cómo citar este artículo}

Barrios-Tao, H., Díaz, V., \& Guerra, Y. M. (2021). Propósitos de la educación frente a desarrollos de inteligencia artificial. Cadernos de Pesquisa, 51, Artículo e07767. https://doi.org/10.1590/198053147767 